\title{
Prevalence and Persistence of Breathing Disorders in Chronic Heart Failure Patients: Preliminary Results from Home Telemonitoring in the HHH Study
}

\author{
GD Pinna ${ }^{1}$, R Maestri ${ }^{1}$, E Gobbi ${ }^{1}$, S Capomolla $^{1}$, C Campana $^{2}$, A Di Lenarda ${ }^{3}$, M Emdin ${ }^{4}$, \\ D Andrews ${ }^{5}$, MT La Rovere ${ }^{1}$, P Johnson $^{5}$, A Mortara ${ }^{6}$, P Sleight $^{5}$ \\ ${ }^{1}$ Fondazione S Maugeri, IRCCS, Montescano, Italy \\ ${ }^{2}$ Policlinico S Matteo, Pavia, Italy \\ ${ }^{3}$ Ospedale Maggiore, Trieste, Italy \\ ${ }^{4}$ Istituto di Fisiologia Clinica, CNR, Pisa, Italy \\ ${ }^{5}$ Oxford University, Oxford, UK \\ ${ }^{6}$ Policlinico di Monza, Monza, Italy
}

\begin{abstract}
In this paper we present preliminary results of the European Community multicountry trial HHH (Home or Hospital in Heart Failure), which assessed the prevalence and persistence of nocturnal breathing disorders in mild-to-moderate CHF patients. All subjects (465) carried out a baseline respiratory recording in the hospital, followed by 12 recordings (one per month) at home. The latter were totally self-managed by the patients, and data were transmitted to the referring hospital through telephone lines. We found that $43 \%$ of the patients had a periodic breathing pattern ( $P B$, waxing and waning of ventilation with or without apneas) during the night lasting $\geq 1$ hour, and the apnea-hypopnea index (AHI) was $\geq 5$ events/hour in $51 \%$ of them. During the 1 year follow-up, a $\mathrm{PB} \geq 1$ hour and an AHI $\geq 5$ events/hour were persistent (i.e., occurred in $>50 \%$ of the recordings) in $43 \%$ and $52 \%$ of the patients. These findings confirm the high prevalence of nocturnal breathing disorders in CHF patients and show that in a large proportion of patients they tend to persist over time.
\end{abstract}

\section{Introduction}

In the last years several studies have highlighted the clinical and pathophysiological relevance of sleep-related breathing disorders in chronic heart failure (CHF) patients, providing evidence that they can have important implications in terms of morbidity, mortality and new treatment strategies [1-4]. Yet, the prevalence of the phenomenon has been investigated only in a few small studies, and it is still not known whether nocturnal breathing disorders represent an occasional phenomenon or tend to persist over time.

Polysomnography is the standard technique used to perform sleep studies and evaluate abnormalities of breathing. This technique, however, is expensive, technically demanding and not widely available. Home telemonitoring of a set of relevant cardiorespiratory signals might constitute a potential alternative, provided the recording and transmission of respiratory data can be carried out by the patient without the need of external specialized support (nurse or technician). We have explored this new strategy in the multi-center multicountry trial HHH (Home or Hospital in Heart failure), a European Community-supported study (contract $\mathrm{n}^{\circ}$ QLG4-CT-2001-02424) aimed, among other goals, at evaluating a new system for long-term home telemonitoring of cardiorespiratory signals, which is totally self-managed by the patient. The study ended successfully on July 2005, providing relevant data to assess both the prevalence and persistence of nocturnal breathing disorders in these subjects. Preliminary results on these two points are presented in this paper.

\section{Methods}

Subjects and protocol

The HHH study has enrolled 465 patients with symptomatic heart failure (NYHA class II-IV), older than 18 years, on optimized medical treatment, with LVEF $\leq 40 \%$ and with at least one episode of decompensation in the previous 12 months. Three countries (UK, Italy and Poland) and 11 cardiological departments (5 in Italy, 3 in UK and 3 in Poland) were involved in the enrolment and follow-up (one year) process.

Subjects were randomized to two groups: i) usual clinical practice and ii) home telemonitoring. Two-third 
of the second group were further randomized to perform a 24-hour cardiorespiratory recording once per month, for a total of 12 recordings. All 465 patients carried out an inhospital baseline cardiorespiratory recording at enrolment in the study.

To record cardiorespiratory signals we used a Holterstyle 24-hour solid-state portable device (Report-24, FM, Monza, Italy). This recorder is suitable to be easily handled by the patient at home since only 3 ECG electrodes are used to pick-up both the ECG signal (single lead) and the respiratory signal (bio-impedance technique), while a small plastic box houses the body movement and position sensors (figure 1). Sampling frequency was $250 \mathrm{~Hz}$ for ECG, $10 \mathrm{~Hz}$ for respiration and $1 \mathrm{~Hz}$ for the movement and position signals.

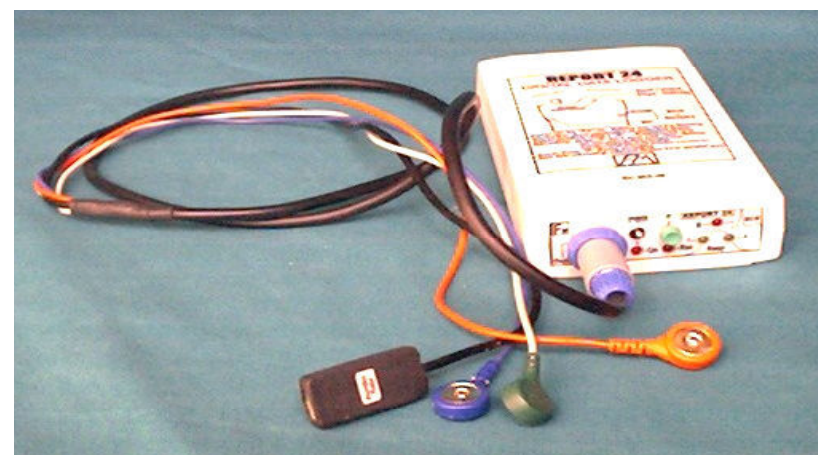

Fig. 1. Picture of the 24-hour cardiorespiratory recorder used in the study (Report-24, FM, Monza, Italy).

After completion of the 24-hour recording, the recorder is connected by the patient to a dedicated modem (Smart Modem, Appel Elettronica, Torino, Italy). Data downloading from the recorder to the Smart Modem's memory buffer and data up-loading from the modem to the receiving station starts automatically after the connection. The overall transmission, including dialing, sending the patient ID and managing all transmission problems, is automatically carried out by the modem without the need of patient intervention.

\section{Signal analysis}

During the study, all received recordings were carefully checked by a signal analyst (one per country), and a request for repetition was issued to the patient in case the poor signal quality.

Respiratory recordings were analyzed using a dedicated software package developed for the study. This software preprocesses the raw respiratory signal to filter out non-respiratory frequency components and remove sudden changes in signal level or occasional spikes. Then an instantaneous tidal volume signal is derived and the respiratory pattern is classified in each consecutive $60 \mathrm{~s}$ segment. The classification includes i) "phasic activity", namely a breathing activity characterized by a regular phasic oscillation of respiration with or without occasional apneas/hypopneas, and ii) "periodic breathing" (PB), defined as a sustained oscillation $(\geq 3 \mathrm{~min}$, modulation depth $\geq 25 \%$,) of ventilatory amplitude characterized by recurring cycles of hyperventilation and hypopnea or apnea. Respiratory segments with poor signal quality were labeled as "not classified" and were excluded from analysis.

For the detection and estimation of hypopneas and apneas, we followed standard criteria used in polysomnography. Accordingly, an hypopnea (apnea) event was defined as a decrease of the instantaneous tidal volume below $50 \%(10 \%)$ of the running tidal volume for more than $10 \mathrm{sec}$.

An interactive procedure allowed the analyst to edit the output of the automatic procedure in order to correct misclassifications and adjust the duration of respiratory events. At the end of this interactive phase, a set of standard and non standard quantitative indexes of breathing disorders was automatically provided by the software, including: i) the duration of $\mathrm{PB}$, ii) the apnea index (AI, number of apneas per hour of recording), iii) the apnea-hypopnea index (AHI, number of apneas and hypopneas per hour of recording), and iv) the apnea prevalence (proportion of time spent in the apnea condition). These parameters were computed for the whole 24-hour period, and for day-time and night-time separately. Night-time was defined as the time while the patient was lying in bed, as indicated by the body position signal, starting from 10.00 p.m. till 8.00 a.m..

By convention, a respiratory recording was considered eligible for the study if the overall duration of good quality signal (i.e., all segments classified as PB or phasic activity) during night-time was $\geq 2.5$ hours. Only data from eligible recordings was entered in the study database.

In order to identify the patients with nocturnal breathing disorders we used different parameters and thresholds. In this preliminary report we present results according to the following classifications: i) overall $\mathrm{PB}$ duration $\geq 1$ hour, ii) AHI $\geq 5$ events /hour, and iii) $\mathrm{AHI} \geq$ 10 events /hour.

A given breathing disorder was defined as "persistent" if it occurred in $>50 \%$ of follow-up recordings, while it was defined as "absent" if it did not occur in any followup recording. Breathing disorders not satisfying these two criteria were defined as "occasional". The persistence study was carried out only in those patients who completed the follow-up and had $\geq 75 \%$ of home recordings eligible.

Descriptive statistics are given as mean \pm SD 


\section{Results}

Prevalence of breathing disorders

Baseline recordings were eligible in 399 patients. Prevalence results according to the defined criteria for the presence of breathing disorders, are given in table 1. It can be seen that, depending on the criterion used, nocturnal breathing disorders affected from one third to more than half of the considered population.

Table 1. Prevalence of nocturnal breathing disorders (from baseline recordings, $\mathrm{N}=399$ )

\begin{tabular}{ccc}
\hline $\begin{array}{c}\text { PB duration } \\
\geq 1 \text { hour }\end{array}$ & $\begin{array}{c}\text { AHI } \\
\geq 5 \text { events/hour }\end{array}$ & $\begin{array}{c}\text { AHI } \\
\geq 10 \text { events/hour }\end{array}$ \\
\hline \hline $43 \%$ & $51 \%$ & $32 \%$ \\
\hline
\end{tabular}

The same results are given in table 2 separately for the 3 countries involved in the study. This table clearly shows that the prevalence of nocturnal breathing disorders was quite homogeneous among different countries.

Table 2. Prevalence of nocturnal breathing in the 3 countries of the study (from baseline recordings)

\begin{tabular}{lccc}
\hline \hline & $\begin{array}{c}\text { PB duration } \\
\geq 1 \text { hour }\end{array}$ & $\begin{array}{c}\text { AHI } \geq 5 \\
\text { events/hour }\end{array}$ & $\begin{array}{c}\text { AHI } \geq 10 \\
\text { events/hour }\end{array}$ \\
\hline \hline Italy & $42 \%$ & $58 \%$ & $37 \%$ \\
Poland & $43 \%$ & $43 \%$ & $28 \%$ \\
UK & $49 \%$ & $53 \%$ & $28 \%$ \\
\hline
\end{tabular}

Feasibility of home recordings

Out of 1689 cardiorespiratory recordings expected to be carried out by the patients at home (being the patient alive, not hospitalized or not dropped out), $89 \%$ were actually performed. Major reasons for not performing were i) refusal (64\%) and ii) organizational problems (29 $\%)$. Almost all (99\%) carried out recordings were received by the analysts located in the country coordinating centers, and about $10 \%$ of them $(\mathrm{N}=152)$ had to be repeated due to poor signal quality. In most cases $(87 \%)$ only one repetition was carried out. Out of 1488 recordings available for analysis, $87 \%$ were eligible for the study. Therefore, the feasibility of home respiratory recording, namely the proportion of expected recordings that were actually carried out and transmitted by the patient and were eligible for the study, was $77 \%$, ranging from $70 \%$ in Poland to $82 \%$ in Italy. The average analyzed time during the night was $6.1 \pm 1.6$ hours.
Persistence of nocturnal breathing disorders

The strict requirements for the assessment of the persistence of nocturnal breathing disorders (see methods) were fulfilled by 75 patients. Representative examples of persistent, absent and not persistent breathing disorder are given respectively in fig. $2 \mathrm{a}, \mathrm{b}, \mathrm{c}$.

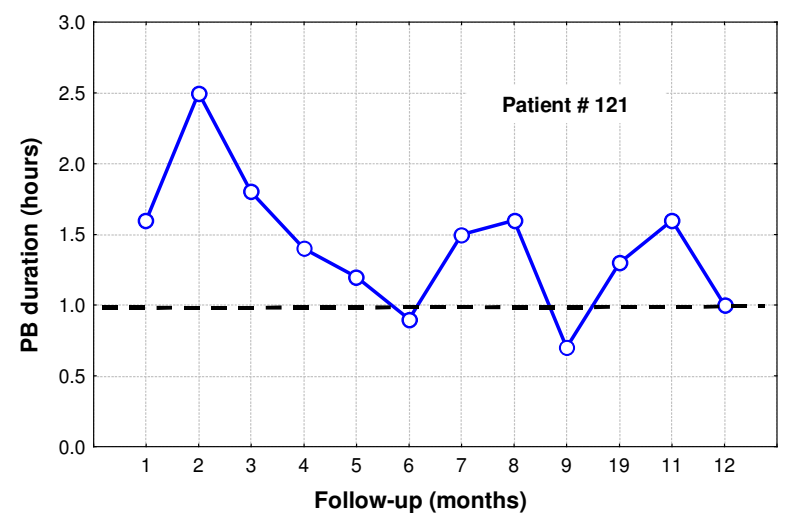

(a)

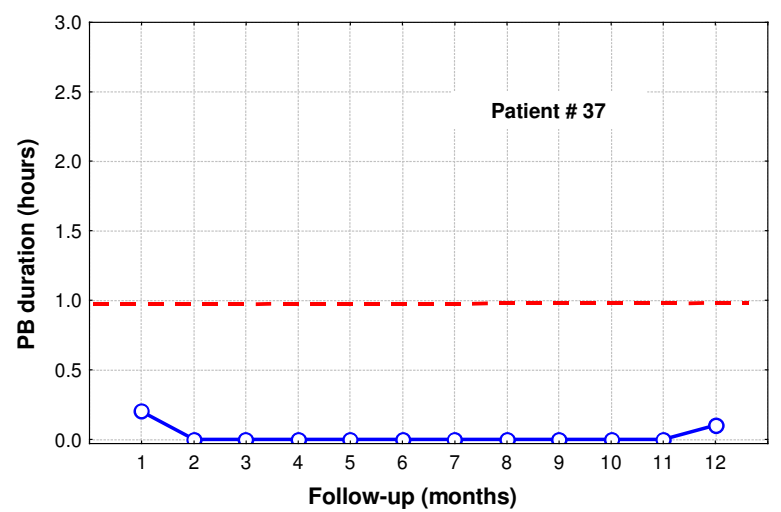

(b)

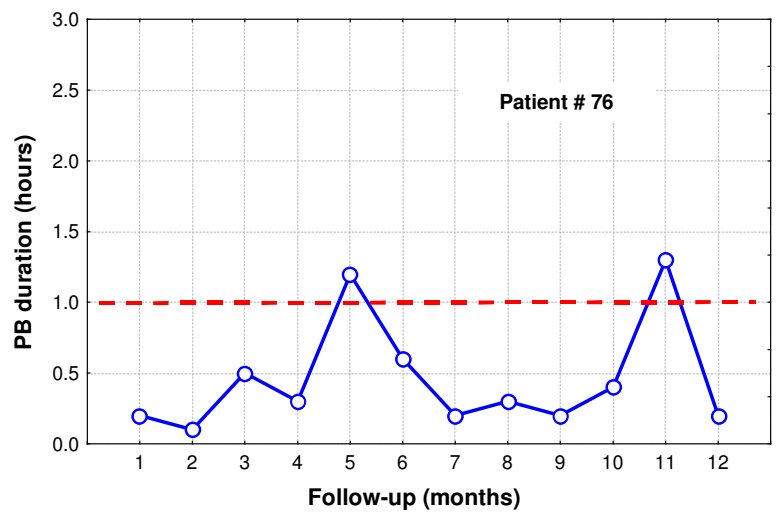

(c)

Fig. 2. Representative examples of persistent (a), absent (b) and occasional (c) breathing disorder according to the criterion of total periodic breathing $(\mathrm{PB})$ duration during the night $\geq 1$ hour 
Global results are reported in table 3. It can be seen that in a great proportion of patients nocturnal breathing disorders persistently occurred during the overall followup (first row of the table), while in only a minority they were permanently absent (second row of the table). In about one third of the patients breathing disorders were only occasional (third row of the table).

Table 3. Persistence of breathing disorders during the 1year follow-up according to 3 different criteria.

\begin{tabular}{lccc}
\hline \hline & $\begin{array}{c}\text { PB duration } \\
\geq 1 \text { hour }\end{array}$ & $\begin{array}{c}\text { AHI } \geq 5 \\
\text { events/hour }\end{array}$ & $\begin{array}{c}\text { AHI } \geq 10 \\
\text { events/hour }\end{array}$ \\
\hline \hline Persistent & $43 \%$ & $52 \%$ & $31 \%$ \\
Absent & $23 \%$ & $16 \%$ & $37 \%$ \\
Occasional & $34 \%$ & $32 \%$ & $32 \%$ \\
\hline
\end{tabular}

\section{Discussion and conclusions}

These preliminary results from the HHH trial clearly show that intermittent long-term home telemonitoring of breathing activity based on self-management of recording and transmitting devices is feasible in CHF patients and their compliance is very high. Using this telemonitoring system we have collected a great amount of valuable information on the prevalence and time course of breathing disorders in these patients, that would not have been possible using traditional polysomnography due to logistic and cost limitations. The key factors determining these achievements were a suitable design of the recording and transmitting devices, which properly balanced accuracy with usability, and a proper training of the patients on the use of telemonitoring devices.

Our findings unambiguously confirm the high prevalence of nocturnal breathing disorders in $\mathrm{CHF}$ patients and, for the first time, provide relevant epidemiological data on the peculiar oscillatory aspect of these disorders, namely periodic breathing.

A novel and relevant finding of this study is that nocturnal breathing disorders, when present, tend to persist over long periods of time (one year) in a great proportion of patients. This has important implications in the clinical management of this patients and may prompt towards more aggressive treatments.

Comparing our results on the prevalence of breathing disorders with those few available in literature [5-7], it appears that our figures of AHI are lower than those previously found by other investigators using standard polysomnography. Besides differences in clinical characteristics of studied populations and in inclusion/exclusion criteria, one major cause of this discrepancy is likely the different procedure we used to compute this index. Indeed, the standard measurement of AHI entails dividing the number of breathing events by the total sleep time, whereas we divided the number of events by the length of the overall analyzed time, roughly corresponding to the total time in bed, which, of course, is much greater. According to a previous study, the ratio between total sleep time and total time in bed is around 0.7 . Correcting our prevalence figures by this factor, we get $73 \%$ and $46 \%$ for, respectively, an AHI $\geq 5$ events/hour and $\mathrm{AHI} \geq 10$ events/hour, which are close to published data.

\section{Acknowledgements}

We would like to express our deep gratitude to all investigators (physicians, nurses, technicians) who have enthusiastically worked in the HHH study, contributing in a crucial way to its success.

\section{References}

[1] Bradley TD, Floras JS. Sleep apnea and heart failure. Part I: obstructive sleep apnea. Circulation 2003; 107: 16711678.

[2] Bradley TD, Floras JS. Sleep apnea and heart failure. Part II: central sleep apnea. Circulation 2003; 107: 1822-1826.

[3] Lanfranchi P, Braghiroli A, Bosimini E, Mazzuero G, Colombo R, Donner CF, Giannuzzi P. Prognostic value of nocturnal Cheyne-Stokes respiration in chronic heart failure patients. Circulation 1999; 99: 1435-1440.

[4] Sin DD, Fitzgerald F, Parker JD, Newton G, Floras JS, Bradley TD. Risk factors for central and obstructive sleep apnea in 450 men and women with congestive heart failure. Am J Respir Crit Care Med 1999; 160: 1101-1106.

[5] Javaheri TS, Parker TJ, Liming JD, Corbett WS, Nishiyama H, Wexler L, Roselle GA. Sleep apnea in 81 ambulatory male patients with stable heart failure. Types and their prevalence, consequences, and presentation. Circulation 1998; 97: 2154-2159.

[6] Mansfeld DR, Solin P, Roebuck T, Bergin P, Kaye DM, Naughton MT. The effect of successful heart transplant treatment of heart failure on central sleep apnea. Chest 2003; 124: 1675-1681.

[7] Roebuck T, Solin P, Kaye DM, Bergin P, Bailey M, Naughton MT. Increased long-term mortality in heart failure due to sleep apnoea is not yet proven. Eur Respir J 2004; 23: 735-740.

Address for correspondence

Gian Domenico Pinna

S. Maugeri Foundation, IRCCS,

Scientific Institute of Montescano, 27040 Montescano (Pavia)

Italy

e-mail: gdpinna@fsm.it 